\title{
Hippocampal microstructural damage correlates with memory impairment in clinically isolated syndrome suggestive of multiple sclerosis
}

Vincent Planche ${ }^{1,2,3}$, Aurélie Ruet ${ }^{1,2,4}$, Pierrick Coupé ${ }^{5}$, Delphine Lamargue-Hamel ${ }^{1,2}$, Mathilde Deloire $^{4}$, Bruno Pereira ${ }^{3}$, José V. Manjon ${ }^{6}$, Fanny Munsch ${ }^{1,2}$, Nicola Moscufo ${ }^{7}$, Dominik S. Meier ${ }^{7}$, Charles R.G. Guttmann ${ }^{1,7}$, Vincent Dousset ${ }^{1,2,4}$, Bruno Brochet ${ }^{1,2,4, *}$, Thomas Tourdias ${ }^{1,2,4, *}$

${ }^{1}$ Univ. Bordeaux, F-33000 Bordeaux, France;

${ }^{2}$ Inserm U1215 - Neurocentre Magendie, F-33000 Bordeaux, France

${ }^{3} \mathrm{CHU}$ de Clermont-Ferrand, F-63000 Clermont-Ferrand, France;

${ }^{4}$ CHU de Bordeaux, F-33000 Bordeaux, France;

${ }^{5}$ Laboratoire Bordelais de Recherche en Informatique, UMR CNRS 5800, F-33405 Talence, France;

${ }^{6}$ Univ. Politécnica de Valencia, 46022 Valencia, Spain

${ }^{7}$ Brigham and Women's Hospital, Harvard Medical School, Boston, MA, United States.

* BB and TT share co-seniorship of this study

Corresponding author: Vincent Planche, Neurocentre Magendie, Inserm U1215, 146 Rue Léo Saignat, 33000, Bordeaux, France; planche.vincent@gmail.com; Phone: +33 (0)5 575737 37; Fax: $+33(0) 557573669$

Key words: multiple sclerosis, clinically isolated syndrome, hippocampus, memory impairment, diffusion tensor imaging

Word count: abstract: 194, article: 3232 (without references)

Tables/Figures: 4 tables, 2 figures

References: 37 


\begin{abstract}
Objective: We investigated whether diffusion tensor imaging (DTI) could reveal early hippocampal damage, and clinically relevant correlates of memory impairment in persons with clinically isolated syndrome (CIS) suggestive of multiple sclerosis (MS).
\end{abstract}

Methods: 37 persons with CIS, 32 with MS and 36 controls prospectively included from 2011 to 2014 were tested for cognitive performances and scanned with 3T-MRI to assess volumetric and DTI changes within the hippocampus, whole brain volume, and T2-lesion load.

Results: While there was no hippocampal atrophy in the CIS group, hippocampal fractional anisotropy (FA) was significantly decreased compared to controls. Decrease in hippocampal FA together with increased mean diffusivity (MD) was even more prominent in MS patients. In CIS, hippocampal MD was correlated with episodic verbal memory performance $(\mathrm{r}=-0.57, \mathrm{p}=0.0002$ and $\mathrm{OR}=0.058$ (95\% $\mathrm{CI}=0.0057-0.59), \mathrm{p}=0.016$ adjusted for age, gender, depression and T2-lesion load), but not with cognitive tasks unrelated to hippocampal functions. Hippocampal MD was the only variable discriminating memory-impaired from memory-preserved persons with $\mathrm{CIS}$ ( $\mathrm{AUC}=0.77$; sensitivity 90.0\%; specificity 70.3\%; PPV 52,9\%; NPV 95,0\%).

Conclusion: DTI alterations within the hippocampus might reflect early neurodegenerative processes that are correlated with episodic memory performance, discriminating persons with CIS according to their memory status. 


\section{Introduction}

Cognitive impairment is frequent in multiple sclerosis (MS) [1,2] and strongly impacts quality of life and vocational status [3]. The two most frequently affected domains are information processing speed (IPS) and episodic memory [1]. While IPS impairment is usually explained by disconnections due to demyelinating lesions and diffuse brain damage [4], several groups have reported that memory impairment could be more specifically related to hippocampal atrophy $[5,6,7,8]$.

Significant memory impairment is already present at the stage of clinically isolated syndrome (CIS) $[9,10]$, the first clinical episode suggestive of MS. However, hippocampal damage is typically not detected at this early stage of MS because atrophy is not yet observed or still confined to few locations such as the thalamus or the striatum $[11,12,13,14]$. To the best of our knowledge, only one study has reported hippocampal atrophy in a CIS group with high T2-lesion load [15]. Therefore, there is a need for imaging biomarkers more sensitive than atrophy, especially at the stage of CIS, which could reflect clinically relevant hippocampal alterations to help the development and validation of future neuroprotective strategies.

Diffusion tensor imaging (DTI) has been efficiently used to reveal widespread white matter damages from the stage of CIS [16] but less consistently within grey matter $[17,18]$. Specifically, there is no report of altered DTI within the hippocampus of persons with CIS and no strong imaging correlate of memory impairment at this stage. Outside the field of MS, DTI has revealed subtle and early hippocampal alterations in Alzheimer's disease [19], but it's currently unknown whether this technique can also be sensitive enough to capture clinically relevant modifications at the very early stage of MS.

In this study, we hypothesized that microstructural damage contributing to early memory impairment could occur in the hippocampus at the stage of CIS and that it might be measurable with DTI while 
there is no hippocampal atrophy. To test this hypothesis, we measured hippocampal volume and DTI metrics within the hippocampus of persons with CIS (PwCIS) to look for correlations with episodic memory scores and to test their ability to discriminate between memory-impaired and memorypreserved PwCIS. A group of healthy subjects and a group of persons with MS (PwMS) were used as negative and positive controls, respectively. 


\section{Methods}

\section{Participants}

All subjects were prospectively enrolled from 2011 to 2014 at our MS center. Written informed consent was obtained prior to participation.

The main population of interest consisted of thirty-seven persons with CIS (PwCIS) enrolled prospectively after approval by the local institutional ethics review board (NCT01865357) between 2 to 6 months after a first clinical event compatible with a demyelinating inflammatory syndrome. At least two clinically silent lesions with $3 \mathrm{~mm}$ minimum diameter were needed for inclusion. One had to be cerebral (ovoid or periventricular), while the other could be located in spine or brain. Exclusion criteria: contraindications to MRI, presence of other neurologic, psychiatric or systemic diseases, steroid treatment within one month, starting or stopping antidepressants or anxiolytic treatments within 2 months of MRI and neuropsychological examination.

We pooled these data with data from a MS study (NCT01207856) to analyze patients with long lasting disease as a positive control. Thirty-two persons with MS (PwMS) diagnosed according to the 2005revised McDonald criteria [20] were included (27 relapsing-remitting, 3 secondary progressive and 2 primary progressive form of MS). Exclusion criteria were dementia (Mini Mental State Examination $<27$ ), other neurologic or psychiatric disorders, drug or alcohol abuse, relapse and/or steroid treatment within 2 months, or severe depression (Beck Depression Inventory $>27$ ).

A total of seventy-six healthy control (HC) subjects, included as negative controls, were free of neurologic, psychiatric, or systemic diseases, and drug or alcohol abuse. Fifty-four subjects were tightly matched for age, sex and educational level to PwCIS, to calculate cognitive z-score (see below). The twenty-two additional subjects were matched for age, sex and educational level to PwMS. 


\section{Neuropsychological testing}

All patients and controls performed the Symbol Digit Modalities Test (SDMT) to assess IPS and the forward digit span subtest of the WAIS-III to assess attention/working memory. In order to assess hippocampal functions (verbal learning and recall), PwCIS and matched controls performed the Selective Reminding Test (SRT) while PwMS and matched controls performed the California Verbal Learning Test (CVLT). Indeed, the SRT was frequently used in previous studies of CIS $[9,10]$ and is part of the brief repeatable battery of neuropsychological testing [21] while the CVLT was the most common validated task used to assess episodic verbal memory in previous MS studies $[6,8]$. The SRTLTS (Long Term Storage) and the CVLT-immediate recall were considered as measures of learning performance, and DR-SRT (Delayed Free Recall) and DR-CVLT as measures of long term recall performance. Each subject also completed a standard questionnaire concerning depressive symptoms (Beck Depression Inventory, BDI).

Each PwCIS or PwMS was compared with the appropriate control group ( $\mathrm{n}=54$ and $\mathrm{n}=22$ respectively), tightly matched for age, sex and educational level to calculate a z-score for each test:

$\mathrm{z}=$ (patient's score - mean value of the matched control group)/standard deviation of the matched control group.

Lower z-score indicates lower performance. A patient was considered impaired in a given cognitive domain if his/her z-score was $<-1.5$, according to agreed-upon threshold [22].

\section{MRI acquisition}

All PwCIs, PwMS and a representative subset of $36 \mathrm{HC}$ were scanned on a $3 \mathrm{~T}$ Philips Achieva MRI system with an 8-channel SENSE head coil. The protocol included a 3D gradient-echo T1-weighted sequence $\left(\mathrm{TR} / \mathrm{TE} / \mathrm{TI} / \mathrm{flip}\right.$ angle $=8.2 \mathrm{~ms} / 3.5 \mathrm{~ms} / 982 \mathrm{~ms} / 7^{\circ}$, resolution $\left.1 \times 1 \times 1 \mathrm{~mm}, 256 \mathrm{~mm} \mathrm{FOV}\right)$ and a $2 \mathrm{D}$ axial fluid attenuated inversion recovery (FLAIR) sequence (TR/TE/TI $=11000 \mathrm{~ms} / 140 \mathrm{~ms} / 2800 \mathrm{~ms}$, 
resolution 1x1.1x3mm, 230mm FOV). A diffusion tensor echo-planar-imaging (EPI) pulse sequence was also acquired: $\mathrm{TR} / \mathrm{TE}=11676 \mathrm{~ms} / 60 \mathrm{~ms}$, $1.6 \times 1.6 \times 1.6 \mathrm{~mm}$ resolution, $230 \mathrm{~mm} \mathrm{FOV}$, with $\mathrm{b}=1000 \mathrm{~s} / \mathrm{mm}^{2}(\Delta=29.4 \mathrm{~ms}, \delta=12.9 \mathrm{~ms})$ in 20 non-co-linear directions and 4 sets of $\mathrm{b}=0 \mathrm{~s} / \mathrm{mm}^{2}$.

\section{MRI analyses}

Lesion load was determined using co-registered T1 and FLAIR sequences and a semi-automated segmentation pipeline based on local thresholding [23] followed by manual correction by a blinded expert (V.P., a neurologist with 4 years of experience).

For volumetric analyses of brain structures, T1-weighted images were processed using the volBrain system (http://volbrain.upv.es). After denoising [24], images were affine-registered [25] into the Montreal Neurological Institute (MNI) space and the total brain volume was estimated. Hippocampus was automatically segmented with a patch-based multi-templates method described in details elsewhere [26] that uses expert manual segmentations in MNI space as priors. Anatomical boundaries of the hippocampus were defined according to the EADC-ADNI Harmonized Protocol [27]. Every mask was then blindly checked and manually corrected (V.P.) for inappropriate inclusion of parahippocampal T1-hypointense lesions, choroidal plexus and/or CSF "pockets". To control for variations in head size, total brain volumes and hippocampal volumes were scaled using the volumetric scaling factor determined through the affine registration to the MNI brain template.

An in-house pipeline (dtiBrain) was used to process diffusion weighted images (DWI). First, images were denoised [28] in order to improve signal-to-noise ratio. Then, head displacement and distortions induced by eddy currents were corrected by performing affine registration [25] of all DWI to the b0 images. The direction table was updated with the estimated motion matrices. To compensate for EPI distortion, a non-rigid registration of DWI to the subject's T1-weighted images in MNI space was 
performed by using ANTs [25]. For that purpose, we found that registration of mean diffusivity map, derived from DWI, to CSF probability map, derived from T1, provided the most accurate strategy by visual inspection of the hippocampal areas. Then, a diffusion tensor model was fitted at each voxel using FSL 5.0 (http://fsl.fmrib.ox.ac.uk/fsl) to measure mean fractional anisotropy (FA) and mean diffusivity (MD) within the hippocampal masks previously generated from T1-weighted images (Fig. $1 \mathrm{~A}, \mathrm{~B}$ and $\mathrm{C})$.

\section{Statistical Analyses}

Right and left hippocampal values were averaged. Statistical analyses were performed with Stata software 12 (StataCorp) and Prism software 6 (Graphpad). The distribution of all continuous data was tested with the Shapiro-Wilk test. We first compared clinical and imaging characteristics between the three groups (HC, CIS, MS) by using the Chi-squared test for categorical variables, and ANOVA or Kruskal-Wallis test (when assumptions of ANOVA were not met) for ordinal variables followed by appropriate post-hoc tests (Tukey-Kramer or Dunn test, respectively), and adjustment on age and gender. If only two groups had to be compared, Student's t-test or Mann-Whitney test was used as appropriate. Cohen's d was used to measure the effect size. Then, relationships between clinical scores and quantitative imaging variables were assessed using correlation coefficients (Pearson or Spearman according to statistical distribution) for CIS and MS groups respectively. The associations were further tested in multivariate context to take into account adjustment on covariables fixed according to univariate results and clinical relevance: age, gender, depression and T2 lesion load. For that purpose, cognitive performances (dependent variables) were predicted with regression models. Linear regression model was used whenever possible while logistic regression was seen as the appropriate alternative for categorical outcome variables by dichotomizing the performances; a log transformation was applied when needed to achieve normality. Considering the issue of multiple 
comparisons, Sidak's correction was used to reduce the risk of type I error. Lastly, Receiver Operating Characteristic (ROC) analysis was performed to test the value of DTI to discriminate between memory-impaired PwCIS (z-score DR-SRT < -1.5) versus those with normal memory performance. All tests were two-tailed, with a type I error set at $\alpha=0.05$. 


\section{Results}

\section{Clinical characteristics and general MRI features}

A total of 37 PwCIS, 32 PwMS and $36 \mathrm{HC}$ assessed with MRI were included (Table 1). Groups were comparable for age, sex ratio and educational level. PwCIS and PwMS showed significantly higher depression scores than controls but they were not significantly different from each other $(p=n s$; $\mathrm{d}=0.39$ ). As expected, EDSS and T2-lesion load were significantly lower in PwCIS than in PwMS. PwCIS presented no global brain atrophy compared to HC whereas PwMS did.

\section{Cognitive assessment}

Median z-scores and percentages of impaired PwCIS and PwMS (z-score $<-1.5$ ) are reported in table 2. Regarding mean or median z-scores, as expected, PwMS performed significantly worse than pwCIS for all the tests. Verbal episodic memory performance was the most frequently affected domain in PwCIS (27.0\%) whereas slowing of IPS was the most frequently affected domain in PwMS (78.1\%).

\section{Hippocampal volume and DTI measures}

Hippocampal volumes normalized for head size were not significantly different between groups $(\mathrm{p}=0.19)$. PwMS showed slightly lower volumes compared to HC even though this difference didn't reach significance (mean volume $9.72 \mathrm{ml}$ and $9.99 \mathrm{ml}$ respectively, d=0.31, Fig.1D). Interestingly, PwCIS and $\mathrm{HC}$ were thoroughly similar for their hippocampal volumes (mean volume $10.06 \mathrm{ml}$ and $9.99 \mathrm{ml}$ respectively, d=0.09, Fig.1D).

Hippocampal FA and MD were significantly different between groups $(p<0.0001$ for both measures, adjusted for age and gender: $\mathrm{FA}=0.171,0.162$ and $0.149 ; \mathrm{MD}=1.269,1.281$ and $1.319 \times 10^{-3} \mathrm{~mm}^{2} / \mathrm{s}$ for HC, CIS and MS groups respectively; Fig. 1E and F). Post-hoc analyses showed that FA was 
significantly decreased in PwCIS compared to $\mathrm{HC}(\mathrm{p}=0.050$, adjusted for age and gender; $\mathrm{d}=0.53)$, as well as in PwMS compared to HC $(\mathrm{p}<0.0001 ; \mathrm{d}=1.05)$ and compared to PwCIS $(\mathrm{p}=0.007 ; \mathrm{d}=0.70)$. In PwMS, MD was significantly increased compared to $\mathrm{HC}(\mathrm{p}<0.0001 ; \mathrm{d}=1.21)$ and compared to PwCIS $(\mathrm{p}=0.004 ; \mathrm{d}=0.65)$. The difference in MD between PwCIS and HC did not reach a statistically significant level ( $\mathrm{p}=0.093 ; \mathrm{d}=0.41)$, but the graphical distribution of hippocampal MD values in the CIS group showed a sub-group with distinctly higher MD values compared to HC.

\section{Correlations between MRI measures and cognitive performance (table 3)}

In the CIS group, in univariate analyses, the hippocampal MD was the only metric strongly correlated with long-term recall $(\mathrm{r}=-0.57, \mathrm{p}=0.0002)$; a correlation that remains significant even after correction for the 32 multiple comparisons run between MRI and clinical data $(p=0.0050)$. By contrast, hippocampal volume and T2-lesion load were not correlated with episodic memory. In multivariate analysis, after adjusting for age, gender, depression and T2-lesion load, the correlation between hippocampal MD and performances on long term recall remained significant with an odds ratio of $0.058(95 \% \mathrm{CI}=0.0057-0.59, \mathrm{p}=0.016)$.

In the MS group, the performances on learning trials were correlated with hippocampal volumes $(\mathrm{r}=0.44, \mathrm{p}=0.01$ and $\beta=1.23,95 \% \mathrm{CI}=0.12-2.34, \mathrm{p}=0.032$ after adjustments $)$. IPS measures were correlated with $\mathrm{T} 2$-lesion load $\left(\mathrm{r}=-0.51, \mathrm{p}=0.004\right.$ and $\beta=-3.5 \times 10^{-5}, 95 \% \mathrm{CI}=-6.8 \times 10^{-5}--3.12 \times 10^{-6}$, $\mathrm{p}=0.033$ after adjustments) but also with both hippocampal FA and MD (respectively $\mathrm{r}=0.36, \mathrm{p}=0.04$, non-significant after adjustments and $\mathrm{r}=-0.52, \mathrm{p}=0.002 ; \beta=-8.95,95 \% \mathrm{CI}=-15.77--2.13, \mathrm{p}=0.012$ after adjustments).

Hippocampal MD discriminates between memory-impaired and memory-preserved PwCIS

Regarding the strong association between hippocampal MD and long-term recall memory 
performances, we tested whether this parameter could discriminate memory-impaired PwCIS from those with normal long-term recall. PwCIS were dichotomized in two subgroups according to their long-term memory performances (i.e. DR-SRT z-score <-1.5). Using ROC analysis we showed that a threshold $\mathrm{MD}$ value $\left(\mathrm{MD}=1.278 \times 10^{-3} \mathrm{~mm}^{2} / \mathrm{s}\right)$ was discriminating these two subgroups with $90.0 \%$ sensitivity, $70.3 \%$ specificity, $52.9 \%$ positive predictive value (PPV) and $95.0 \%$ negative predictive value $(\mathrm{NPV}$ ) (area under the curve $=0.77,95 \% \mathrm{CI}=0.62-0.92, \mathrm{p}=0.013$, Fig. 2A). Hippocampal MD was the only demographical, clinical or radiological characteristic significantly different between memory-impaired and memory-preserved PwCIS ( $\mathrm{p}=0.011$, Fig. 2B and table 4). 


\section{Discussion}

In this study, hippocampal microstructural alterations measured by DTI appear to be already present in the early stages of MS (PwCIS), while no significant hippocampal or global brain atrophy compared to $\mathrm{HC}$ is evident. Furthermore, increased hippocampal MD was associated with early memory impairment in these PwCIS.

A quarter of our population of PwCIS showed episodic verbal memory impairment in learning and retrieval tasks which is consistent with previous studies using the same test $[9,10]$. Interestingly, memory impairment was more frequent than slowing of IPS in PwCIS. This observation suggests that a neurodegenerative process starts early within the hippocampus contributing to memory decline, even at a stage where IPS is relatively preserved because complete disconnection phenomena due to widespread demyelination are generally lower at the CIS stage than later on [4]. Furthermore, our PwCIS were not treated with disease modifying therapies, avoiding bias linked to heterogeneous therapeutic response.

Our data support the concept that early memory deficit in MS could be related to specific hippocampal vulnerability, independent of the extent of focal white matter lesions. Hippocampal FA was significantly decreased in the CIS group and even more in the MS group, in which a significant increase of hippocampal MD was also observed. In previous work assessing PwCIS, deep grey matter DTI changes were detected, while no hippocampal DTI changes were apparent [17]. However, the sensitivity to DTI changes in the hippocampus might have been hampered by partial voluming effects due to a larger voxel size $(3.33 \mathrm{~mm} \times 3.33 \mathrm{~mm} \times 3.00 \mathrm{~mm})$ [17], compared with our $1.6 \mathrm{~mm}^{3}$ voxel size. We also believe that applying a denoising procedure further increased our sensitivity towards microstructural alterations detected by DTI, enabling our findings [28]. 
In support of the clinical relevance of our finding of early hippocampal damage in PwCIS, we were able to show a strong and independent correlation of hippocampal MD with a hippocampus-dependent task (DR-SRT), but not with cognitive tasks that are not reliant on hippocampal function (i.e, IPS and attention/working memory, even though some authors argue for a continuum between working memory and long term memory encoding [29]). Hippocampal MD in PwCIS was correlated to hippocampal-dependent long-term recall in the absence of significant hippocampal volume loss, suggesting that hippocampal MD reflects an earlier pathophysiological process that likely precedes atrophy. Interestingly, and despite smaller differences between groups, the increase in MD appears to be more clinically relevant than the decrease in FA in the explored context. MD might be more stable and reproducible than FA in the hippocampus (i.e. smaller dispersion of the values, even in the control group), which can be reasonably expected in such a grey matter structure where diffusion tends to be more isotropic and therefore imperfectly represented by FA as a measure of fiber orientation. Therefore either FA and MD measure different underlying histological substrate or, more likely, FA is less reliable than MD in grey matter which is in line with previous results [16,17]. Higher MD might correspond to a mixture of hippocampal demyelination, neuronal or synaptic loss together with inflammatory cells as described in post-mortem studies of MS brains [30].

We also showed that hippocampal MD discriminates memory-impaired from memory-preserved patients with a sensitivity of $90 \%$, a specificity of $70.3 \%$ and a NPV of $95.0 \%$. Among clinical and other MRI criteria, hippocampal MD was the only predictor of memory impairment in this group. Therefore, stabilization of low hippocampal MD might be an interesting candidate endpoint (surrogate marker with a very good NPV) in future proof-of-concept phase II clinical trials in CIS and early MS. Unambiguous monitoring of cognitive performances in the context of a neuroprotective agent in clinical trials is complicated by the length of the cognitive testing, the test/retest effect and 
confounding factors such as participation of the subject, fatigue or depression [31]. In this context, stabilization of low hippocampal MD might provide a complementary and unbiased endpoint. DTI of the hippocampus might also help to improve patient selection by stratifying those with and without hippocampal damage, therefore powering future neuroprotective trials by including more homogeneous population in comparison groups.

In contrast, in PwMS, episodic memory correlated with hippocampal volume, but the correlation between hippocampal MD and memory scores was not significant. This finding suggests that at a more advanced stage, assessing the microstructure of the hippocampus with DTI might no longer be sensitive and relevant. Furthermore, T2-lesion load and hippocampal DTI metrics were correlated with IPS scores. These findings can suggest that hippocampus is involved in IPS but also that hippocampal DTI at the stage of clinically definite MS reflects more general brain damage and in turn correlate better with IPS that has been associated to global cerebral damage [2]. This is consistent with data in the literature showing that cognitive impairment is associated with several imaging metrics in more advanced MS [32] because cognitive impairment is probably the result of a complex interplay between several alterations at this stage [33].

We acknowledge an important shortcoming of this meta-analysis of our data, namely that the cognitive testing was different for the CIS and MS groups, limiting our ability to directly compare PwCIS to PwMS. Nevertheless, a previous paper reported no difference in sensitivity between SRT and CVLT [34] and furthermore, we believe that the insights gained by separately comparing these groups of patients to matched $\mathrm{HC}$ explored with the same tests maintain full validity. We also acknowledge that the sample size of each group is relatively small and therefore these results can be viewed as preliminary and need confirmation in a larger cohort of PwCIS. Another limitation is that we didn't acquire 3D-double inversion recovery MRI, limiting our ability to detect focal hippocampal lesions 
[35]. Therefore, our DTI findings might reflect focal, as well as more diffuse alterations in the hippocampus. Because the PPV was only $52.9 \%$, it will be of great interest for future studies to assess whether PwCIS with increased hippocampal MD but preserved memory functions display compensatory mechanisms measured by hippocampal activation and functional connectivity, as demonstrated in PwMS [36]. Similarly, longitudinal data should identify whether those patients are likely to show memory alterations in long-term follow-up. DTI could also be combined with susceptibility weighted imaging that could also reveal subtle alterations because it has been demonstrated that iron deposition is present within the hippocampus at the stage of CIS [37].

In conclusion, episodic memory impairment was correlated with hippocampal DTI changes at the stage of CIS, in the absence of a significant effect of global lesion volume or atrophy on any of the cognitive measures. This argues for a clinically relevant neurodegenerative process occurring in the hippocampus in the early stages of MS, and suggests that DTI measurement in this structure might provide a sensitive indicator of early neurodegenerative changes. 


Controls with
MRI $(\mathrm{n}=36)$$\quad$ CIS $(\mathrm{n}=37) \quad$ MS $(\mathrm{n}=32) \quad$ p-value

\section{Clinical features}

\begin{tabular}{|c|c|c|c|c|}
\hline Mean age, years [range] & $37.8[21-60]$ & $37.4[19-59]$ & $41.3[28-50]$ & 0.18 \\
\hline Sex ratio $(F / M)$ & $24 / 12$ & $29 / 8$ & $23 / 9$ & 0.53 \\
\hline Mean disease duration, months [range] & - & $<6$ & $107[12-216]$ & $<0.0001$ \\
\hline Education level $\left(\mathrm{High}_{\mathrm{Low}}{ }^{\S}\right)$ & $29 / 7$ & $24 / 13$ & $19 / 13$ & 0.14 \\
\hline Median EDSS score [range] & - & $1.0[0-6]$ & $3.0[0-8]$ & $<0.0001$ \\
\hline Median depression (BDI) score [range] & $3.5[0-13]$ & $9.0^{* *}[0-36]$ & $14.0^{* * *}[0-26]$ & $<0.0001$ \\
\hline \multicolumn{5}{|l|}{ Imaging features } \\
\hline Median T2 lesion volume, $\mathrm{ml}[$ range] & - & $\begin{array}{c}2.11 \\
{[0.18-60.28]}\end{array}$ & $\begin{array}{c}8.38 \\
{[1.25-65.69]}\end{array}$ & 0.001 \\
\hline Median normalized brain volume, $\mathrm{ml}$ [range] & $\begin{array}{c}1564 \\
{[1475-1676)}\end{array}$ & $\begin{array}{c}1570 \\
{[1335-1657]}\end{array}$ & $\begin{array}{c}1485^{* * *} \\
{[1276-1667]}\end{array}$ & 0.0002 \\
\hline
\end{tabular}

Table 1: Clinical and general MRI features. BDI = Beck Depression Inventory; CIS = Clinically Isolated Syndrome; MS = Multiple Sclerosis; EDSS = Expanded Disability Status Scale. $\S$ Education level was considered as high or low according to French baccalaureate (equivalent to A-level). ** $\mathrm{p}<0.01$ and $* * * \mathrm{p}<0.001$, patients $v s$ controls. 


\begin{tabular}{ccccc}
\hline & \multicolumn{2}{c}{ CIS $(\mathrm{n}=37)$} & \multicolumn{1}{c}{ MS $(\mathrm{n}=32)$} \\
\cline { 2 - 5 } & $\begin{array}{c}\text { Impaired } \\
\text { patients (\%) }\end{array}$ & Median z-score [range] & $\begin{array}{c}\text { Impaired } \\
\text { patients (\%) }\end{array}$ & Median z-score [range] \\
\hline Information processing speed & 13.9 & $-0.628[-3.78-+1.74]$ & 78.1 & $-2.217[-4.48-+0.17]$ \\
Attention/Working memory & 18.9 & $-0.525[-1.50-+1.43]$ & 21.9 & $-1.324[-2.45-+2.06]$ \\
$\quad$ Episodic verbal memory & & & & \\
$\quad$ - learning trials & 27.0 & $-0.269[-5.27-+1.21]$ & 65.6 & $-3.234[-9.43-+0.90]$ \\
- long term recall & 27.0 & $+0.611[-9.16-+0.61]$ & 62.5 & $-1.767[-7.45-+0.67]$ \\
\hline
\end{tabular}

Table 2: Neuropsychological performances of persons with CIS and persons with MS. CIS = Clinically Isolated Syndrome; MS = Multiple Sclerosis. The threshold to dichotomize impaired/non impaired patients was set at z-score $<-1.5$ (z-scores were computed relative to appropriate control groups for CIS and MS, see method). 


\begin{tabular}{|c|c|c|c|c|}
\hline CIS $(n=37)$ & $\begin{array}{c}\text { T2 } \\
\text { Lesion load }\end{array}$ & $\begin{array}{l}\text { Hippocampal } \\
\text { Volume }\end{array}$ & $\begin{array}{c}\text { Hippocampal } \\
\text { FA } \\
\end{array}$ & $\begin{array}{c}\text { Hippocampal } \\
\text { MD }\end{array}$ \\
\hline Information processing speed & $\mathrm{r}=-0.13, \mathrm{p}=0.45$ & $\mathrm{r}=0.04, \mathrm{p}=0.84$ & $\mathrm{r}=0.10 \mathrm{p}=0.54$ & $\mathrm{r}=-0.23, \mathrm{p}=0.18$ \\
\hline Attention/Working memory & $\mathrm{r}=-0.16, \mathrm{p}=0.36$ & $\mathrm{r}=-0.11, \mathrm{p}=0.53$ & $\mathrm{r}=-0.17, \mathrm{p}=0.31$ & $\mathrm{r}=-0.01, \mathrm{p}=0.98$ \\
\hline Episodic verbal memory (learning trials) & $\mathrm{r}=-0.24, \mathrm{p}=0.14$ & $\mathrm{r}=0.02, \mathrm{p}=0.93$ & $\mathrm{r}=-0.07, \mathrm{p}=0.67$ & $\mathrm{r}=-0.29, \mathrm{p}=0.08$ \\
\hline Episodic verbal memory (long term recall) & $\mathrm{r}=-0.07, \mathrm{p}=0.68$ & $\mathrm{r}=0.02, \mathrm{p}=0.89$ & $\mathrm{r}=0.01, \mathrm{p}=0.96$ & $\begin{array}{c}r=-0.57, p=0.0002 \\
(p=0.016)^{\#}\end{array}$ \\
\hline MS $(n=32)$ & $\begin{array}{c}\text { T2 } \\
\text { Lesion load }\end{array}$ & $\begin{array}{c}\text { Hippocampal } \\
\text { Volume } \\
\end{array}$ & $\begin{array}{c}\text { Hippocampal } \\
\text { FA } \\
\end{array}$ & $\begin{array}{c}\text { Hippocampal } \\
\text { MD }\end{array}$ \\
\hline Information processing speed & $\begin{array}{c}r=-0.51, p=0.004 \\
(p=0.033)^{\dagger}\end{array}$ & $\mathrm{r}=0.25, \mathrm{p}=0.18$ & $\begin{array}{c}\mathbf{r}=\mathbf{0 . 3 6}, \mathbf{p}=\mathbf{0 . 0 4} \\
(\text { n.s. })^{\#}\end{array}$ & $\begin{array}{c}r=-0.52, p=0.002 \\
(p=0.012)^{\#}\end{array}$ \\
\hline Attention/Working memory & $\mathrm{r}=-0.16, \mathrm{p}=0.38$ & $\mathrm{r}=0.17, \mathrm{p}=0.35$ & $\mathrm{r}=0.16, \mathrm{p}=0.40$ & $\mathrm{r}=-0.05, \mathrm{p}=0.76$ \\
\hline Episodic verbal memory (learning trials) & $\mathrm{r}=-0.15, \mathrm{p}=0.41$ & $\begin{array}{c}r=0.44, p=0.01 \\
(p=0.032)^{\#}\end{array}$ & $\mathrm{r}=0.19, \mathrm{p}=0.31$ & $\mathrm{r}=-0.30, \mathrm{p}=0.09$ \\
\hline Episodic verbal memory (long term recall) & $\mathrm{r}=-0.10, \mathrm{p}=0.60$ & $\mathrm{r}=0.29, \mathrm{p}=0.10$ & $\mathrm{r}=0.12, \mathrm{p}=0.52$ & $\mathrm{r}=-0.12, \mathrm{p}=0.52$ \\
\hline
\end{tabular}

Table 3: Correlations between MRI measures and neuropsychological z-scores. CIS = Clinically Isolated Syndrome; FA = Fractional Anisotropy; $\mathrm{MD}=$ Mean Diffusivity; $\mathrm{MS}=$ Multiple Sclerosis. \#, adjusted on age, gender, depression and T2-lesion load; †, adjusted on age, gender and depression. To explore multiple leads, $\mathrm{p}$ values of univariate correlations were uncorrected for multiple comparisons in this table. 


\begin{tabular}{|c|c|c|c|}
\hline CIS & $\begin{array}{l}\text { Memory-impaired } \\
(\mathrm{n}=10)\end{array}$ & $\begin{array}{l}\text { Memory-preserved } \\
(\mathrm{n}=27)\end{array}$ & p-value \\
\hline Median memory z-score [range] & $-2.44[-9.16--1.83]$ & $0.61[-0.61-0.61]$ & $<0.0001$ \\
\hline Mean age, years [range] & $37.5[19-59]$ & $37.3[23-58]$ & 0.96 \\
\hline $\operatorname{Sex}(F / M)$ & $6 / 4$ & $23 / 4$ & 0.17 \\
\hline Educational level (High/Low) & $5 / 5$ & $19 / 8$ & 0.27 \\
\hline Median T2 lesion volume, $\mathrm{ml}$ [range] & $2.05[0.24-15.54]$ & $1.80[0.18-60.28]$ & 0.89 \\
\hline Median normalized brain volume, $\mathrm{ml}$ [range] & $1546[1454-1647]$ & $1587[1335-1657]$ & 0.33 \\
\hline $\begin{array}{c}\text { Median normalized hippocampal volume, } \mathrm{ml} \\
\text { [range] }\end{array}$ & $10.4[8.16-10.86]$ & $10.2[8.73-11.79]$ & 0.72 \\
\hline Median hippocampal FA [range] & $\begin{array}{c}0.1650 \\
{[0.1471-0.1695]}\end{array}$ & $\begin{array}{c}0.1577 \\
{[0.1429-0.1986]}\end{array}$ & 0.69 \\
\hline Median hippocampal MD, $\times 10^{-3} \mathrm{~mm}^{2} / \mathrm{s}$ [range] & $\begin{array}{c}1.301 \\
{[1.258-1.379]}\end{array}$ & $\begin{array}{c}1.250 \\
{[1.208-1.469]}\end{array}$ & 0.011 \\
\hline
\end{tabular}

Table 4: Clinical and MRI characteristics of memory-impaired vs memory-preserved patients with

CIS. CIS = Clinically Isolated Syndrome; FA = Fractional Anisotropy; MD = Mean Diffusivity. 


\section{Figures captions}

Figure 1: Hippocampal volumes and DTI measures in patients and controls.

A-C Examples of MRI from a patient with MS. A. T1-weighted images were used for hippocampi segmentation (cyan lines). B. Color-coded FA maps superimposed to T1-weighted images. C. FA maps registered in the MNI space with the same hippocampal masks. D. Plots of the normalized hippocampal volumes of the three groups. E. Plots of the fractional anisotropy values within the hippocampal masks of the three groups. F. Plots of the mean diffusivity values within the hippocampal masks of the three groups. Each point is an individual measure and bars are mean and standard error of the mean. CIS $=$ Clinically Isolated Syndrome; MS $=$ Multiple Sclerosis. ${ }^{*} p<0.05,{ }^{* *} p<0.01$, $* * * \mathrm{p}<0.001$ adjusted on age and gender.

Figure 2: Hippocampal MD discriminates between memory-impaired and memory-preserved persons with CIS.

A. ROC curve defined a threshold MD value $\left(1.278 \times 10^{-3} \mathrm{~mm}^{2} / \mathrm{s}\right.$, red dashed line $)$ that classified memory-impaired and memory-preserved patients (i.e. patients with DR-SRT z-score $<1.5$ ) with a sensitivity of $90.0 \%$ and a specificity of $70.3 \%$. B. Plots of mean diffusivity values in memory impaired and memory-preserved patients with CIS. ${ }^{*} p=0.011$. AUC $=$ Area Under the Curve; CIS $=$ Clinically Isolated Syndrome; DR-SRT = Delayed Recall-Selective Reminding Test. 

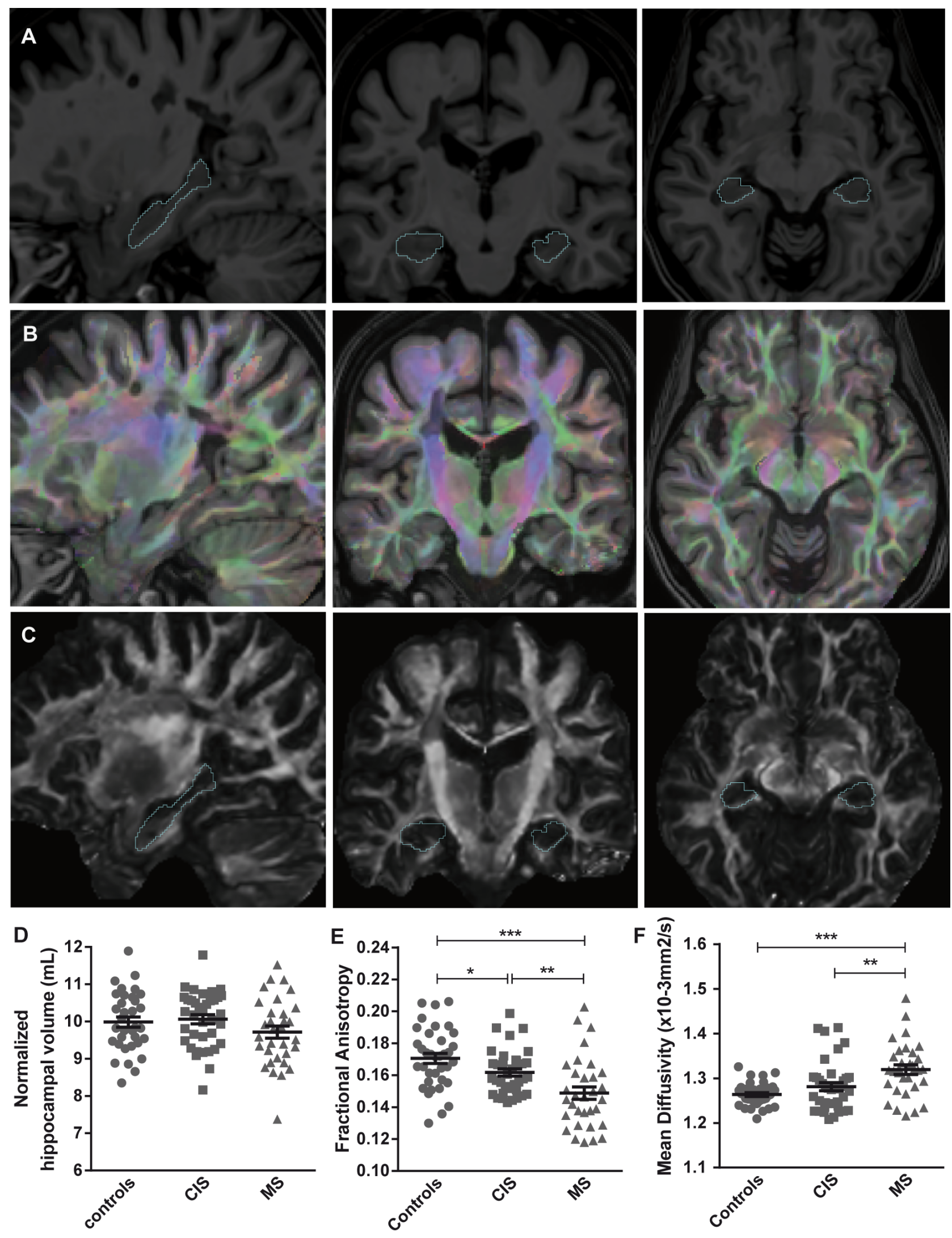
A

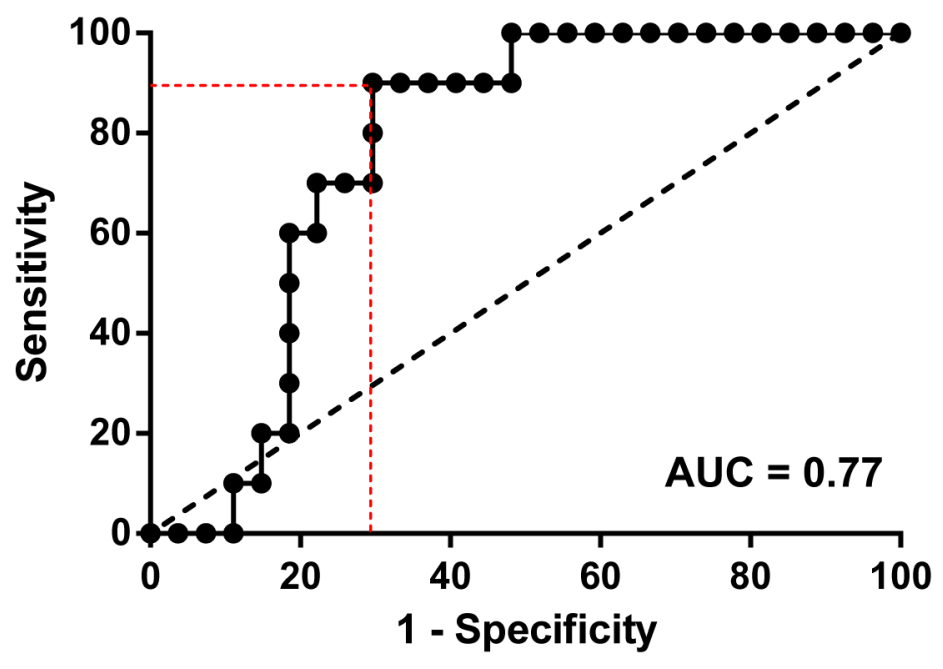

B

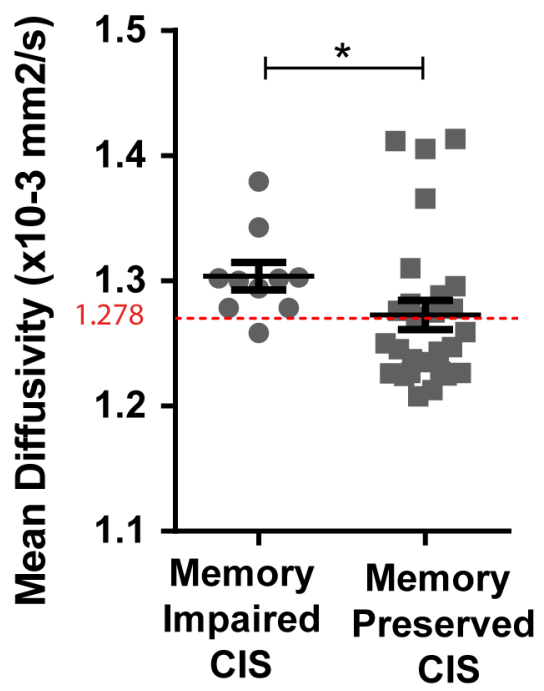




\section{Study funding}

This study was supported by the ARSEP Fondation, Bordeaux University Hospital and TEVA and Merck-Sereno laboratories. The work was further supported by public grants from the French Agence Nationale de la Recherche within the context of the Investments for the Future program referenced ANR-10-LABX-57 named TRAIL (project IBIO-NI and HR-DTI) and ANR-10-LABX-43 named BRAIN (Project MEMO-MS). This study has also been carried out with financial support from the "Investments for the future" Programme IdEx Bordeaux - CPU (ANR-10-IDEX-03-02) and the CNRS multidisciplinary project "Défi ImagIn" HL-MRI. The sponsors did not participate in any aspect of the design or performance of the study, including data collection, management, analysis, and interpretation or the preparation, review, and approval of the manuscript.

\section{Disclosures}

VP received travel expenses from ARSEP Fondation, Biogen, Teva-Lundbeck and Merk-Serono. AR received research grants and/or consulting fees from Novartis, Biogen, Merck-Serono, Bayer Healthcare, Roche, Teva, and Genzyme unrelated to the submitted work. BB serves on scientific advisory boards for and has received honoraria or research support for its institution from Biogen-idec, Merck-Serono, Novartis, Genzyme, Teva, Roche, Medday and Bayer outside the submitted work. CRGG is the recipient of a grant from Sanofi, and holds shares in Roche, Novartis, GSK, Alnylam, Protalix, Agenus, Arrowhead, and Cocrystal.

\section{Acknowledgments}

The authors thank Julie Charré-Morin and Aurore Saubusse (Bordeaux University Hospital) for neuropsychological testing. 


\section{References}

1. Chiaravalloti ND, DeLuca J. Cognitive impairment in multiple sclerosis. Lancet Neurol. 2008; 7(12):1139-1151.

2. Rocca MA, Amato MP, De Stefano N, et al. Clinical and imaging assessment of cognitive dysfunction in multiple sclerosis. Lancet Neurol. 2015; 14(3):302-317.

3. Ruet A, Deloire M, Hamel D, et al. Cognitive impairment, health-related quality of life and vocational status at early stages of multiple sclerosis: a 7-year longitudinal study. J. Neurol. 2013; 260(3):776-784.

4. Dineen RA, Vilisaar J, Hlinka J, et al. Disconnection as a mechanism for cognitive dysfunction in multiple sclerosis. Brain. 2009; 132(Pt 1):239-249.

5. Sicotte NL, Kern KC, Giesser BS, et al. Regional hippocampal atrophy in multiple sclerosis. Brain. 2008; 131(Pt 4):1134-1141.

6. Benedict RHB, Ramasamy D, Munschauer F, Weinstock-Guttman B, Zivadinov R. Memory impairment in multiple sclerosis: correlation with deep grey matter and mesial temporal atrophy. $J$. Neurol. Neurosurg. Psychiatry. 2009; 80(2):201-206.

7. Longoni G, Rocca MA, Pagani E, et al. Deficits in memory and visuospatial learning correlate with regional hippocampal atrophy in MS. Brain Struct. Funct. 2015; 220(1):435-444.

8. Koenig KA, Sakaie KE, Lowe MJ, et al. Hippocampal volume is related to cognitive decline and fornicial diffusion measures in multiple sclerosis. Magn. Reson. Imaging. 2014; 32(4):354-358.

9. Achiron A, Barak Y. Cognitive impairment in probable multiple sclerosis. J. Neurol. Neurosurg. Psychiatry. 2003; 74(4):443-446.

10. Feuillet L, Reuter F, Audoin B, et al. Early cognitive impairment in patients with clinically isolated syndrome suggestive of multiple sclerosis. Mult. Scler. 2007; 13(1):124-127.

11. Henry RG, Shieh M, Okuda DT, et al. Regional grey matter atrophy in clinically isolated syndromes at presentation. J. Neurol. Neurosurg. Psychiatry. 2008; 79(11):1236-1244. 
12. Rocca MA, Preziosa P, Mesaros S, et al. Clinically Isolated Syndrome Suggestive of Multiple Sclerosis: Dynamic Patterns of Gray and White Matter Changes-A 2-year MR Imaging Study. Radiology. 2016; 278(3):841-853.

13. Calabrese M, Rinaldi F, Mattisi I, et al. The predictive value of gray matter atrophy in clinically isolated syndromes. Neurology. 2011; 77(3):257-263.

14. Audoin B, Zaaraoui W, Reuter F, et al. Atrophy mainly affects the limbic system and the deep grey matter at the first stage of multiple sclerosis. J. Neurol. Neurosurg. Psychiatry. 2010; 81(6):690-695.

15. Bergsland N, Horakova D, Dwyer MG, et al. Subcortical and cortical gray matter atrophy in a large sample of patients with clinically isolated syndrome and early relapsing-remitting multiple sclerosis. AJNR Am. J. Neuroradiol. 2012; 33(8):1573-1578.

16. Preziosa P, Rocca MA, Mesaros S, et al. Intrinsic damage to the major white matter tracts in patients with different clinical phenotypes of multiple sclerosis: a voxelwise diffusion-tensor MR study. Radiology. 2011; 260(2):541-550.

17. Cappellani R, Bergsland N, Weinstock-Guttman B, et al. Diffusion tensor MRI alterations of subcortical deep gray matter in clinically isolated syndrome. J. Neurol. Sci. 2014; 338(1-2):128-134.

18. Deppe M, Krämer J, Tenberge J-G, et al. Early silent microstructural degeneration and atrophy of the thalamocortical network in multiple sclerosis. Hum. Brain Mapp. 2016.

19. Douaud G, Menke RAL, Gass A, et al. Brain microstructure reveals early abnormalities more than two years prior to clinical progression from mild cognitive impairment to Alzheimer's disease. $J$. Neurosci. 2013; 33(5):2147-2155.

20. Polman CH, Reingold SC, Edan G, et al. Diagnostic criteria for multiple sclerosis: 2005 revisions to the 'McDonald Criteria'. Ann. Neurol. 2005; 58(6):840-846.

21. Boringa JB, Lazeron RH, Reuling IE, et al. The brief repeatable battery of neuropsychological tests: normative values allow application in multiple sclerosis clinical practice. Mult. Scler. 2001; 7(4):263-267.

22. Benedict RHB, Zivadinov R. Reliability and validity of neuropsychological screening and 
assessment strategies in MS. J. Neurol. 2007; 254 Supp1 2:II22-II25.

23. Van Leemput K, Maes F, Vandermeulen D, Colchester A, Suetens P. Automated segmentation of multiple sclerosis lesions by model outlier detection. IEEE Trans. Med. Imaging. 2001; 20(8):677688.

24. Manjón JV, Coupé P, Martí-Bonmatí L, Collins DL, Robles M. Adaptive non-local means denoising of MR images with spatially varying noise levels. J. Magn. Reson. Imaging. 2010; 31(1):192-203.

25. Avants BB, Tustison NJ, Song G, et al. A reproducible evaluation of ANTs similarity metric performance in brain image registration. NeuroImage. 2011; 54(3):2033-2044.

26. Coupé P, Manjón JV, Fonov V, et al. Patch-based segmentation using expert priors: application to hippocampus and ventricle segmentation. NeuroImage. 2011; 54(2):940-954.

27. Frisoni GB, Jack CR, Bocchetta M, et al. The EADC-ADNI Harmonized Protocol for manual hippocampal segmentation on magnetic resonance: Evidence of validity. Alzheimers Dement. 2015; 11(2):111-125.

28. Manjón JV, Coupé P, Concha L, et al. Diffusion weighted image denoising using overcomplete local PCA. PloS One. 2013; 8(9):e73021.

29. Leszczynski M. How does hippocampus contribute to working memory processing? Front. Hum. Neurosci. 2011; 5:168.

30. Dutta R, Chang A, Doud MK, et al. Demyelination causes synaptic alterations in hippocampi from multiple sclerosis patients. Ann. Neurol. 2011; 69(3):445-454.

31. Benedict RHB, Zivadinov R. Risk factors for and management of cognitive dysfunction in multiple sclerosis. Nat. Rev. Neurol. 2011; 7(6):332-342.

32. Daams M, Steenwijk MD, Schoonheim MM, et al. Multi-parametric structural magnetic resonance imaging in relation to cognitive dysfunction in long-standing multiple sclerosis. Mult. Scler. 2016; 22(5):608-619.

33. Preziosa P, Rocca MA, Pagani E, et al. Structural MRI correlates of cognitive impairment in 
patients with multiple sclerosis: A Multicenter Study. Hum. Brain Mapp. 2016; 37(4):1627-1644.

34. Strober L, Englert J, Munschauer F, et al. Sensitivity of conventional memory tests in multiple sclerosis: comparing the Rao Brief Repeatable Neuropsychological Battery and the Minimal Assessment of Cognitive Function in MS. Mult. Scler. 2009; 15(9):1077-1084.

35. Calabrese M, Battaglini M, Giorgio A, et al. Imaging distribution and frequency of cortical lesions in patients with multiple sclerosis. Neurology. 2010; 75(14):1234-1240.

36. Hulst HE, Schoonheim MM, Van Geest Q, et al. Memory impairment in multiple sclerosis: Relevance of hippocampal activation and hippocampal connectivity. Mult. Scler. 2015.

37. Hagemeier J, Weinstock-Guttman B, Bergsland N, et al. Iron deposition on SWI-filtered phase in the subcortical deep gray matter of patients with clinically isolated syndrome may precede structurespecific atrophy. AJNR Am. J. Neuroradiol. 2012; 33(8):1596-1601. 